Journal of Mathematics and Statistics 6 (1): 34-41, 2010

ISSN 1549-3644

(C) 2010 Science Publications

\title{
Some Properties of Certain Subclass of Meromorphically Multivalent Functions Defined By Linear Operator
}

\author{
F. Ghanim and M. Darus \\ School of Mathematical Sciences, Faculty of Science and Technology, \\ University Kebangsaan Malaysia, Bangi 43600 Selangor D. Ehsan, Malaysia
}

\begin{abstract}
Problem statement: By means of the Hadamard product (or convolution), a linear operator was introduced. This operator was motivated by many authors namely Srivastava, Swaminathan, Owa and many others. The operator was indeed needed to create new ideas in the area of geometric function theory. Approach: The linear operator of meromorphic p-valent functions was proposed and defined. The preliminary concept of subordination was introduced to give sharp proofs for certain sufficient conditions of the linear operator aforementioned. Results: Having the operator, subordination theorems established by using standard concept of subordination and reduced to well-known results studied by various authors. The operator was then applied for fractional calculus and obtained new subordination theorem. Conclusion: Therefore, interesting operators could be obtained with some earlier results and standard methods.
\end{abstract}

Key words: Hypergeometric, meromorphic, hadamard product, linear operator

\section{INTRODUCTION}

Let $\sum_{\mathrm{p}}$ denote the class of meromorphic functions $\mathrm{f}(\mathrm{z})$ normalized by:

$$
f(z)=\frac{1}{z^{p}}+\sum_{n=0}^{\infty} a_{n+p} z^{n+p}
$$

which are analytic and p-valent in the punctured unit disk $U=\{z: 0<|z|<1\}$. For $0 \leq \beta<p$, we denote by $S_{p}^{*}(\beta)$ and $\mathrm{K}_{\mathrm{p}}(\mathrm{p})$ the subclasses of $\sum_{\mathrm{p}}$ consisting of all meromorphic functions which are, respectively, starlike of order $\beta$ and convex of order $\beta$ inU.

The classes $S_{p}^{*}(\beta), K_{p}(p)$ and various other subclasses of $\sum_{\mathrm{p}}$ have been studied rather extensively by (Aouf, 1993; Aouf and Hossen, 1993; Aouf and Srivastava, 1997; Ghanim and Darus, 2009a; 2009b; Joshi and Srivastava, 1999; Kukarni et al., 1998; Mogra, 1990a; 1990b; Owa et al., 1997; Srivastava and Owa, 1992; Uralegaddi and Somanatha, 1992; Yang, 1995).

For functions $f_{j}(z)(j=1,2)$ defined by:

$$
f_{j}(z)=\frac{1}{z^{p}}+\sum_{n=0}^{\infty} a_{n+p, j} z^{n+p}
$$

we denote the Hadamard product (or convolution) of $\mathrm{f}_{1}(\mathrm{z})$ and $\mathrm{f}_{2}(\mathrm{z})$ by:

$$
\left(\mathrm{f}_{1} * \mathrm{f}_{2}\right)=\frac{1}{\mathrm{z}^{\mathrm{p}}}+\sum_{\mathrm{n}=0}^{\infty} \mathrm{a}_{\mathrm{n}+\mathrm{p}, 1} \mathrm{a}_{\mathrm{n}+\mathrm{p}, 2} \mathrm{z}^{\mathrm{n}+\mathrm{p}}
$$

Let us define the function $\phi_{\mathrm{p}}(\alpha, \beta ; \mathrm{z})$ by:

$\phi_{p}(\alpha, \beta ; z)=\frac{1}{z^{p}}+\sum_{n=0}^{\infty} \frac{(\alpha)_{n+1}}{(\beta)_{n+1}} z^{n+1-1}$

$(\beta \neq 0,-1,-2, \ldots$ and $\alpha \in \mathbb{C} \backslash\{0\}, p \in N)$.

where $(\lambda) n$ is the Pochhammer symbol. We note that:

$$
\phi_{\mathrm{p}}(\alpha, \beta ; \mathrm{z})=\frac{1}{\mathrm{z}^{\mathrm{p}}}{ }_{2} \mathrm{~F}_{1}(1, \alpha, \beta ; \mathrm{z})
$$

Where:

$$
{ }_{2} F_{1}(1, \alpha, \beta ; z)=\sum_{n=0}^{\infty} \frac{(1)_{n+1}(\alpha)_{n+1}}{(\beta)_{n+1}} \frac{z^{n}}{n !}
$$

is the well-known Gaussian hypergeometric function. Corresponding to the function $\phi_{\mathrm{p}}(\alpha, \beta ; \mathrm{z})$ using the Hadamard product for $\mathrm{f}(\mathrm{z}) \in \sum_{\mathrm{p}}$, we define a new linear operator $\mathrm{L}_{\mathrm{p}}(\alpha, \beta)$ on $\sum_{\mathrm{p}}$ :

$$
\begin{aligned}
& \mathrm{L}_{\mathrm{p}}(\alpha, \beta) \mathrm{f}(\mathrm{z})=\phi_{\mathrm{p}}(\alpha, \beta ; \mathrm{z}) * \mathrm{f}(\mathrm{z}) \\
& =\frac{1}{\mathrm{z}^{\mathrm{p}}}+\sum_{\mathrm{n}=0}^{\infty}\left|\frac{(\alpha)_{\mathrm{n}+2}}{(\beta)_{\mathrm{n}+2}}\right| \mathrm{a}_{\mathrm{n}+\mathrm{p}} \mathrm{z}^{\mathrm{n}+\mathrm{p}}
\end{aligned}
$$

Corresponding Author: M. Darus, School of Mathematical Sciences, Faculty of Science and Technology, University Kebangsaan Malaysia, Bangi 43600 Selangor D. Ehsan, Malaysia 
The meromorphic functions with the generalized hypergeometric functions were considered recently by (Cho and Kim, 2007; Dziok and Srivastava, 2002; 2003; Liu, 2003; Liu and Srivastava, 2001; 2004a; 2004b).

Also for a function $\mathrm{f}(\mathrm{z}) \in \sum_{\mathrm{p}}$, we define the integral operator $\mathrm{J}_{v, p}$ by:

$$
J_{v, p}=\frac{v}{z^{v+p}} \int_{0}^{z} t^{v+p-1} f(t) d t, \quad v>0
$$

There are many researches (Uralegaddi and Somanatha, 1991; Whittaker and Watson, 1927; Yang, $1995 ; 1996)$ in which the operator $J_{v, p}$ was investigated.

For a function $f \in L_{p}(\alpha, \beta) f(z)$ we define:

$$
\mathrm{I}_{0}\left(\mathrm{~L}_{\mathrm{p}}(\alpha, \beta) \mathrm{f}(\mathrm{z})\right)=\mathrm{L}_{\mathrm{p}}(\alpha, \beta) \mathrm{f}(\mathrm{z})
$$

and for $\mathrm{k}=1,2,3, \ldots$

$$
\begin{aligned}
& I^{k}\left(L_{p}(\alpha, \beta) f(z)\right)=z\left(I^{k-1} L_{p}(\alpha, \beta) f(z)\right)^{\prime}+\frac{1+p}{z} \\
& =\frac{1}{z^{p}}+\sum_{n=0}^{\infty}(n+p)^{k}\left|\frac{(\alpha)_{n+2}}{(\beta)_{n+2}}\right| a_{n+p} z^{n+p}
\end{aligned}
$$

We note that $\mathrm{I}^{\mathrm{k}}\left(\mathrm{L}_{1}(\alpha, \beta) \mathrm{f}(\mathrm{z})\right)$ studied by Ghanim and Darus (2009a; 2009b).

Also, it follows from (5) that (Liu, 2003):

$$
z\left(L_{p}(\alpha, \beta) f(z)\right)^{\prime}=\alpha L_{p}(\alpha+1, \beta) f(z)-(\alpha+p) L_{p}(\alpha, \beta)
$$

and

$$
\begin{aligned}
& z\left(I^{k} L_{p}(\alpha, \beta) f(z)\right)^{\prime} \\
& =\alpha I^{k} L_{p}(\alpha+1, \beta) f(z)-(\alpha+p) I^{k} L_{p}(\alpha, \beta)
\end{aligned}
$$

We denote by $\sum_{\mathrm{p}}(\mathrm{k}, \gamma, \delta, \mu, \lambda)$ the class of all functions $\mathrm{f}(\mathrm{z}) \in \sum_{\mathrm{p}}$ such that:

$$
\begin{aligned}
& \Re\left\{(1-\lambda)\left(\frac{I^{k} L(\alpha, \beta) f(z)}{I^{k} L(\alpha, \beta) g(z)}\right)^{\mu}\right. \\
& \left.+\lambda\left(\frac{I^{k} L(\alpha+1, \beta) f(z)}{I^{k} L(\alpha+1, \beta) g(z)}\right) \cdot\left(\frac{I^{k} L(\alpha, \beta) f(z)}{I^{k} L(\alpha, \beta) g(z)}\right)^{\mu-1}\right\}>\gamma
\end{aligned}
$$

where, $g(z) \in \sum_{p}$ satisfies the following condition:
$\Re\left\{\frac{I^{k} L(\alpha, \beta) g(z)}{I^{k} L(\alpha+1, \beta) g(z)}\right\}>\delta, \quad(0 \leq \delta<1, z \in U)$

where, $\gamma$ and $\mu$ are real numbers such that $0 \leq \gamma\langle 1, \mu\rangle$ 0 and $\lambda \in \mathbb{C}$ with $\Re\{\lambda\}>0$.

\section{MATERIALS AND METHODS}

To establish our main results we need the following lemmas.

Lemma A: Let $\Omega$ be a set in the complex plane $\mathbb{C}$ and let the function $\Psi: \mathbb{C}^{2} \rightarrow \mathbb{C}$ satisfy the condition $\Psi\left(\mathrm{ir}_{2}\right.$, $\left.\mathrm{s}_{1}\right) \notin \Omega$ for all real $\mathrm{r}_{2}, \mathrm{~s}_{1} \leq \frac{1+\mathrm{r}_{2}^{2}}{2}$. If $\mathrm{q}(\mathrm{z})$ is analytic in $\mathrm{U}$ with $\mathrm{q}(0)=1$ and $\Psi\left(\mathrm{q}(\mathrm{z}), \quad \mathrm{zq}^{\prime}(\mathrm{z})\right) \in \Omega, \in \mathrm{U}$ then

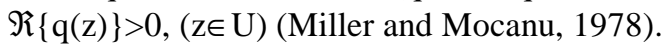

Lemma B: If $\mathrm{q}(\mathrm{z})$ is analytic in $\mathrm{U}$ with $\mathrm{q}(0)=1$ and if $\lambda \in \mathbb{C} \backslash\{0\}$ with $\Re\{\lambda\}>0$ then $\Re\left\{\mathrm{q}(\mathrm{z})+\lambda \mathrm{zq}^{\prime}(\mathrm{z})\right\}>\gamma,(0$ $\leq \gamma<1)$ implies $\mathfrak{R}\{\mathrm{q}(\mathrm{z})\}>\gamma(1-\gamma)(2 \xi-1)$, where $\xi$ is given by:

$$
\xi=\xi(\Re \lambda)=\int_{0}^{1}\left(1+t^{9 \Re \lambda}\right)^{-1} d t
$$

which is increasing function of $\Re\{\lambda\}$ and $\frac{1}{2} \leq \xi<1$. The estimate is sharp in the sense that the bound cannot be improved (Ponnusamy, 1995).

For real or complex numbers $a, b, c,\left(c \notin z_{0}^{-}\right)$, the Gauss hypergeometric function is defined by:

$$
{ }_{2} \mathrm{~F}_{1}(\alpha, \beta, \gamma ; \mathrm{z})=1+\frac{\alpha \beta}{\gamma} \frac{\mathrm{z}}{1 !}+\frac{\alpha(\alpha+1) \beta(\beta+1)}{\gamma(\gamma+1)} \frac{\mathrm{z}^{2}}{2 !}+\ldots
$$

We note that the above series converges absolutely for $z \in U$ and hence represents an analytic function in the unit disc U (Whittaker and Watson, 1927).

Each of the identities (asserted by Lemma C) is fairly well known (Liu and Srivastava, 2004b; Whittaker and Watson, 1927).

Lemma C: For real or complex parameters $\mathrm{a}, \mathrm{b}, \mathrm{c},\left(\mathrm{c} \notin \mathrm{z}_{0}^{-}\right)$:

$$
\begin{aligned}
& \int_{0}^{1} \mathrm{t}^{\mathrm{b}-1}(1-\mathrm{t})^{\mathrm{c}-\mathrm{b}-1}(1-\mathrm{tz})^{-\mathrm{a}} \mathrm{dt} \\
& =\frac{\Gamma(\beta) \Gamma(\gamma-\beta)}{\Gamma(\gamma)}{ }_{2} \mathrm{~F}_{1}(\alpha, \beta, \gamma ; \mathrm{z})
\end{aligned}
$$


$(\Re(c)>\Re(b)>0):$

$$
{ }_{2} \mathrm{~F}_{1}(\alpha, \beta, \gamma ; \mathrm{z})=(1-\mathrm{z}){ }_{2}^{-\mathrm{a}} \mathrm{F}_{1}\left(\alpha, \gamma-\beta, \gamma ; \frac{\mathrm{z}}{\mathrm{z}-1}\right) ;
$$$$
{ }_{2} \mathrm{~F}_{1}(\alpha, \beta, \gamma ; \mathrm{z})={ }_{2} \mathrm{~F}_{1}(\beta, \gamma, \gamma ; \mathrm{z})
$$

and

$$
{ }_{2} \mathrm{~F}_{1}\left(1,1,2 ; \frac{1}{2}\right)=2 \ln 2
$$

\section{RESULTS}

Theorem 1: Let $f \in \sum_{p}(k, \gamma, \delta, \mu, \lambda), a \in R \backslash\{0\}$ and $\lambda \geq 0$. Then:

$$
\Re\left(\frac{I^{k} L(\alpha, \beta) g(z)}{I^{k} L(\alpha, \beta) g(z)}\right)>\frac{2 \alpha \gamma \mu+\delta \lambda}{2 \alpha \mu+\delta \lambda}
$$

$(0 \leq \gamma<1, \quad \mu>0, \quad z \in U)$ where the function $g(z) \in \sum_{p}$ satisfies the condition (10).

Proof: Let $\xi=\frac{2 \alpha \gamma \mu+\delta \lambda}{2 \alpha \mu+\delta \lambda}$ and we define the function q(z) by:

$$
q(z)=\frac{1}{(1-\xi)}\left\{\left(\frac{I^{k} L(\alpha, \beta) f(z)}{I^{k} L(\alpha, \beta) g(z)}\right)^{\mu}-\xi\right\}
$$

Then $\mathrm{q}(\mathrm{z})$ is analytic in $\mathrm{U}$ and $\mathrm{q}(0)=1$. If we set:

$$
h(z)=\frac{I^{k} L(\alpha, \beta) g(z)}{I^{k} L(\alpha+1, \beta) g(z)}
$$

then by the hypothesis $\mathfrak{R}\{\mathrm{h}(\mathrm{z})\}>\delta$. Differentiating (14) and using the identity (8), we have:

$$
\begin{aligned}
& (1-\lambda)\left(\frac{I^{k} L(\alpha, \beta) f(z)}{I^{k} L(\alpha, \beta) g(z)}\right)^{\mu} \\
& +\lambda \frac{I^{k} L(\alpha+1, \beta) f(z)}{I^{k} L(\alpha+1, \beta) g(z)}\left(\frac{I^{k} L(\alpha, \beta) f(z)}{I^{k} L(\alpha, \beta) g(z)}\right)^{\mu-1} \\
& =[\xi+(1-\xi) q(z)]+\frac{\lambda(1-\xi)}{\alpha \mu} h(z) z q^{\prime}(z)
\end{aligned}
$$

Let us define the function $\Psi(\mathrm{r}, \mathrm{s})$ by:

$$
\Psi(\mathrm{r}, \mathrm{s})=\xi+(1-\xi) \mathrm{r}+\frac{\lambda(1-\xi)}{\alpha \mu} \mathrm{h}(\mathrm{z}) \mathrm{s}
$$
obtain:

Using (17) and the fact that $\mathrm{f} \in \sum_{\mathrm{p}}(\mathrm{k}, \gamma, \delta, \mu, \lambda)$, we

$$
\left\{\Psi\left(\mathrm{q}(\mathrm{z}), \mathrm{zq}^{\prime}(\mathrm{z})\right) ; \mathrm{z} \in \mathrm{U}\right\} \subset \Omega=\{\mathrm{w} \in \mathbb{C}: \Re(\mathrm{w})>\gamma\}
$$

Now for all real $\mathrm{r}_{2}, \mathrm{~s}_{1} \leq \frac{1+\mathrm{r}_{2}^{2}}{2}$, we have:

$$
\begin{aligned}
& \Re\left\{\Psi\left(\mathrm{ir}_{2}, \mathrm{~s}_{1}\right)\right\}=\xi+\frac{\lambda(1-\xi)}{\alpha \mu} \Re\{\mathrm{h}(\mathrm{z})\} \\
& \leq \xi-\frac{\lambda \delta(1-\xi)\left(1+\mathrm{r}_{2}^{2}\right)}{2 \alpha \mu} \leq \xi-\frac{\lambda \delta(1-\xi)}{2 \alpha \mu}=\gamma
\end{aligned}
$$

Hence for each $\mathrm{z} \in \mathrm{U}, \Psi\left(\mathrm{ir}_{2}, \mathrm{~s}_{1}\right) \notin \Omega$. Thus by Lemma A, we have $\Re\{\mathrm{q}(\mathrm{z})\}>0$ and hence:

$$
\Re\left(\frac{I^{k} L(\alpha, \beta) f(z)}{I^{k} L(\alpha, \beta) g(z)}\right)^{\mu}>\xi, \quad z \in U
$$

This proves Theorem 1.

Corollary 1: Let the functions $\mathrm{f}(\mathrm{z})$ and $\mathrm{g}(\mathrm{z})$ be in $\sum_{\mathrm{p}}$ and let $\mathrm{g}(\mathrm{z})$ satisfy the condition (10). If $\mathrm{a} \in \mathrm{R} \backslash\{0\}, \lambda \geq 1$ and:

$$
\Re\left\{(1-\lambda) \frac{\mathrm{I}^{\mathrm{k}} \mathrm{L}(\alpha, \beta) \mathrm{f}(\mathrm{z})}{\mathrm{I}^{\mathrm{k}} \mathrm{L}(\alpha, \beta) \mathrm{g}(\mathrm{z})}+\lambda \frac{\mathrm{I}^{\mathrm{k}} \mathrm{L}(\alpha+1, \beta) \mathrm{f}(\mathrm{z})}{\mathrm{I}^{\mathrm{k}} \mathrm{L}(\alpha+1, \beta) \mathrm{g}(\mathrm{z})}\right\}>\gamma
$$

$0 \leq \gamma<1, \mathrm{z} \in \mathrm{U}$. Then:

$\Re\left\{\frac{\mathrm{I}^{\mathrm{L}} \mathrm{L}(\alpha+1, \beta) \mathrm{f}(\mathrm{z})}{\mathrm{I}^{k} \mathrm{~L}(\alpha+1, \beta) \mathrm{g}(\mathrm{z})}\right\}>\xi=\frac{\gamma(2 \alpha+\delta)+\delta(\lambda-1)}{2 \alpha+\lambda \delta}$

Proof: We have:

$$
\begin{aligned}
& \lambda \frac{I^{k} L(\alpha+1, \beta) f(z)}{I^{k} L(\alpha+1, \beta) g(z)} \\
& =\left[(1-\lambda) \frac{I^{k} L(\alpha, \beta) f(z)}{I^{k} L(\alpha, \beta) g(z)}+\lambda \frac{I^{k} L(\alpha+1, \beta) f(z)}{I^{k} L(\alpha+1, \beta) g(z)}\right] \\
& +(\lambda-1) \frac{I^{k} L(\alpha, \beta) f(z)}{I^{k} L(\alpha, \beta) g(z)}
\end{aligned}
$$

Since $\lambda>1$ making use of (18) and (14) (for $\mu=1$ ), we deduce that:

$\Re\left\{\frac{\mathrm{I}^{\mathrm{L}} \mathrm{L}(\alpha+1, \beta) \mathrm{f}(\mathrm{z})}{\mathrm{I}^{k} \mathrm{~L}(\alpha+1, \beta) \mathrm{g}(\mathrm{z})}\right\}>\xi=\frac{\gamma(2 \alpha+\delta)+\delta(\lambda-1)}{2 \alpha+\lambda \delta}$ 
Corollary 2: Let $\lambda \in \mathbb{C} \backslash\{0\}$ with $\Re\{\lambda\}>0$ and $\mathrm{a} \in \mathrm{R} \backslash\{0\}$. If $\mathrm{f}(\mathrm{z}) \in \sum_{\mathrm{p}}$ satisfies the following condition:

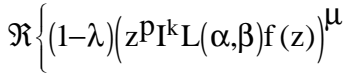

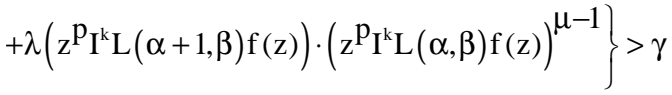

$$
\begin{aligned}
& (0 \leq \gamma<1, \mu>0, p \in N, z \in U)
\end{aligned}
$$

then

$$
\Re\left\{z^{\mathrm{p}} \mathrm{I}^{\mathrm{k}} \mathrm{L}(\alpha, \beta) \mathrm{f}(\mathrm{z})\right\}^{\mu}>\frac{2 \mu \alpha \gamma+\Re\{\lambda\}}{2 \mu \alpha+\Re\{\lambda\}}
$$

Further, if $\lambda \geq 1 ; a \in R\{0\}$ and $\mathrm{f}(\mathrm{z}) \in \sum_{\mathrm{p}}$ satisfies $\Re\left\{(1-\lambda) \mathrm{z}^{\mathrm{p}} \mathrm{I}^{\mathrm{k}} \mathrm{L}(\alpha, \beta) \mathrm{f}(\mathrm{z})+\lambda\left(\mathrm{z}^{\mathrm{p}} \mathrm{I}^{\mathrm{k}} \mathrm{L}(\alpha+1, \beta) \mathrm{f}(\mathrm{z})\right)\right\}>\gamma$. Then:

$$
\begin{aligned}
& \Re\left\{z^{\mathrm{p}} \mathrm{I}^{\mathrm{k}} \mathrm{L}(\alpha+1, \beta) \mathrm{f}(\mathrm{z}\}>\frac{(2 \mathrm{a}+1) \gamma+\lambda-1}{2 \mathrm{a}+\lambda},\right. \\
& (0 \leq \gamma<1, \mathrm{p} \in \mathrm{N}, \mathrm{z} \in \mathrm{U})
\end{aligned}
$$

Proof: The results (20) and (21) follow by putting $\mathrm{g}(\mathrm{z})=\frac{1}{\mathrm{z}^{\mathrm{p}}}$ in Theorem 1 and Corollary 1 , respectively.

Theorem 2: Let $\lambda \in \mathbb{C} \backslash\{0\}$ with and $a \in R \backslash\{0\}$. If $\mathrm{f}(\mathrm{z}) \in \sum_{\mathrm{p}}$ satisfies the following condition:

$$
\begin{aligned}
& \Re\left\{(1-\lambda)\left(z^{p} I^{k} L(\alpha, \beta) f(z)\right)^{\mu}\right. \\
& \left.+\lambda\left(z^{p} I^{k} L(\alpha+1, \beta) f(z)\right) \cdot\left(z^{p} I^{k} L(\alpha, \beta) f(z)\right)^{\mu-1}\right\} \\
& >\gamma(0 \leq \gamma<1, \mu>0, p \in N, z \in U)
\end{aligned}
$$

Then:

$$
\Re\left\{z^{p} I^{k} L(\alpha, \beta) f(z)\right\}^{\mu}>\gamma+(1-\gamma)(2 \rho-1)
$$

Where:

$\rho=\frac{1}{2}{ }_{2} \mathrm{~F}_{1}\left(1,1, \frac{\mu \mathrm{a}}{\Re\{\lambda\}}, \frac{1}{2}\right), \quad \mathrm{z} \in \mathrm{U}$

Proof: Let:

$$
q(z)=\left(z^{p} I^{k} L(\alpha, \beta) f(z)\right)^{\mu}
$$

Then $\mathrm{q}(\mathrm{z})$ is analytic in $U$ with $\mathrm{q}(0)=1$. Differentiating $q(\mathrm{z})$ with respect to $\mathrm{z}$ and using the identity (8), we obtain:

$$
\begin{aligned}
& (1-\lambda)\left(z^{\mathrm{p}} \mathrm{I}^{\mathrm{k}} \mathrm{L}(\alpha, \beta) \mathrm{f}(\mathrm{z})\right)^{\mu} \\
& +\lambda\left(\mathrm{z}^{\mathrm{p}} \mathrm{I}^{\mathrm{k}} \mathrm{L}(\alpha+1, \beta) \mathrm{f}(\mathrm{z})\right) \cdot\left(\mathrm{z}^{\mathrm{p}} \mathrm{I}^{\mathrm{k}} \mathrm{L}(\alpha, \beta) \mathrm{f}(\mathrm{z})\right)^{\mu-1} \\
& =\mathrm{q}(\mathrm{z})+\frac{\lambda \mathrm{zq}(\mathrm{z})}{\mu \mathrm{a}}
\end{aligned}
$$

So that by the hypothesis (22), we have:

$$
\Re\left\{\mathrm{q}(\mathrm{z})+\frac{\lambda \mathrm{zq}^{\prime}(\mathrm{z})}{\mu \mathrm{a}}\right\}>\gamma
$$

In view of Lemma $B$, this implies that:

$$
\Re\{q(z)\}>\gamma+(1-\gamma)(2 p-1)
$$

Where:

$$
\rho=\rho(\Re\{\lambda\})=\int_{0}^{1}\left(1+\mathrm{t}^{\frac{\Re\{\lambda\}}{\mu \mathrm{a}}}\right)^{-1} \mathrm{dt}
$$

Putting $\Re\{\lambda\}=\lambda_{1}>0$, we have:

$$
\rho=\int_{0}^{1}\left(1+t^{\frac{\Re\{\lambda\}}{\mu \alpha}}\right)^{-1} d t=\frac{\mu \alpha}{\lambda_{1}} \int_{0}^{1} u^{\frac{\mu \alpha}{\lambda_{1}}-1}(1+u)^{-1} d u
$$

Using (11)-(13), we obtain:

$$
\rho={ }_{2} \mathrm{~F}_{1}\left(1, \frac{\mu \alpha}{\lambda_{1}} ; \frac{\mu \alpha}{\lambda_{1}}+1 ;-1\right)=\frac{1}{2}{ }_{2} \mathrm{~F}_{1}\left(1,1, \frac{\alpha}{\lambda}+1, \frac{1}{2}\right)
$$

Thus the proof of Theorem 2 is complete.

Corollary 3: Let $\lambda \in \Re, \mu=1$ with $\lambda \geq 1$. If $\mathrm{f}(\mathrm{z}) \in \sum_{\mathrm{p}}$ satisfies:

$$
\begin{aligned}
& \Re\left\{(1-\lambda)\left(z^{\mathrm{p}} \mathrm{I}^{\mathrm{k}} \mathrm{L}(\alpha, \beta) \mathrm{f}(\mathrm{z})\right)\right. \\
& \left.+\lambda\left(\mathrm{z}^{\mathrm{p}} \mathrm{I}^{\mathrm{k}} \mathrm{L}(\alpha+1, \beta) \mathrm{f}(\mathrm{z})\right)\right\}>\gamma
\end{aligned}
$$

$(0 \leq \gamma<1, \alpha \in \mathrm{R} \backslash\{0\}, \mathrm{p} \in \mathrm{N}, \mathrm{z} \in \mathrm{U})$, then:

$$
\Re\left\{\left(z^{\mathrm{p}} \mathrm{I}^{\mathrm{k}} \mathrm{L}(\alpha+1, \beta) \mathrm{f}(\mathrm{z})\right)\right\}>\gamma+(1-\gamma)\left(2 \rho^{*}-1\right)\left(1-\lambda^{-1}\right)
$$


Where:

$$
\rho^{*}=\frac{1}{2}{ }_{2} \mathrm{~F}_{1}\left(1,1, \frac{\alpha}{\lambda}+1, \frac{1}{2}\right)
$$

Proof: The result follows by using the identity:

$$
\begin{aligned}
& \lambda z^{p} I^{k} L(\alpha+1, \beta) f(z) \\
& =\left[(1-\lambda)\left(z^{p} I^{k} L(\alpha, \beta) f(z)\right)+\lambda\left(z^{p} I^{k} L(\alpha+1, \beta) f(z)\right)\right] \\
& +(\lambda-1)\left(z^{p} I^{k} L(\alpha, \beta) f(z)\right)
\end{aligned}
$$

In the following theorem, we shall extend the above results as follows.

Theorem 3: Suppose that the functions $\mathrm{f}(\mathrm{z})$ and $\mathrm{g}(\mathrm{z})$ are in $\sum_{\mathrm{p}}$ and suppose $\mathrm{g}(\mathrm{z})$ satisfies the condition (10). If:

$$
\begin{aligned}
& \Re\left\{\frac{I^{k} L(\alpha+1, \beta) f(z)}{I^{k} L(\alpha+1, \beta) g(z)}-\frac{I^{k} L(\alpha, \beta) f(z)}{I^{k} L(\alpha, \beta) g(z)}\right\}>\frac{(1-\gamma) \delta}{2 \alpha} \\
& (0 \leq \gamma<1, \alpha \in R \backslash\{0\}, 0 \leq \delta<1, z \in U), \text { then } \\
& \Re\left\{\frac{I^{k} L(\alpha, \beta) f(z)}{I^{k} L(\alpha+1, \beta) g(z)}\right\}>\gamma
\end{aligned}
$$

and

$$
\Re\left\{\frac{\mathrm{I}^{k} \mathrm{~L}(\alpha+1, \beta) \mathrm{f}(\mathrm{z})}{\mathrm{I}^{k} \mathrm{~L}(\alpha+1, \beta) \mathrm{g}(\mathrm{z})}\right\}>\frac{(2 \alpha+1+\delta) \gamma-\delta}{2 \alpha}
$$

Proof: Let:

$$
\mathrm{q}(\mathrm{z})=\frac{1}{(1-\gamma)}\left\{\frac{\mathrm{I}^{\mathrm{k}} \mathrm{L}(\alpha, \beta) \mathrm{f}(\mathrm{z})}{\mathrm{I}^{\mathrm{k}} \mathrm{L}(\alpha, \beta) \mathrm{g}(\mathrm{z})}-\gamma\right\}
$$

Then $\mathrm{q}(\mathrm{z})$ is analytic in $\mathrm{U}$ with $\mathrm{q}(0)=1$. Putting:

$$
\Phi(\mathrm{z})=\frac{\mathrm{I}^{\mathrm{k}} \mathrm{L}(\alpha, \beta) \mathrm{g}(\mathrm{z})}{\mathrm{I}^{\mathrm{k}} \mathrm{L}(\alpha+1, \beta) \mathrm{g}(\mathrm{z})}
$$

We observe that by hypothesis, $\Re\{\Phi(\mathrm{z})\}>\delta$, in $\mathrm{U}$. A simple computation shows that:

$$
\begin{aligned}
& \frac{(1-\gamma) \mathrm{zq}^{\prime}(\mathrm{z}) \Re\{\Phi\}}{\alpha}=\frac{\mathrm{I}^{\mathrm{k}} \mathrm{L}(\alpha+1, \beta) \mathrm{f}(\mathrm{z})}{\mathrm{I}^{\mathrm{k}} \mathrm{L}(\alpha+1, \beta) \mathrm{g}(\mathrm{z})} \\
& -\frac{\mathrm{I}^{\mathrm{k}} \mathrm{L}(\alpha, \beta) \mathrm{f}(\mathrm{z})}{\mathrm{I}^{\mathrm{k}} \mathrm{L}(\alpha, \beta) \mathrm{g}(\mathrm{z})}=\Psi\left(\mathrm{q}(\mathrm{z}), \mathrm{zq}^{\prime}(\mathrm{z})\right)
\end{aligned}
$$

Where:

$$
\Psi(\mathrm{r}, \mathrm{s})=\frac{(1-\gamma) \Phi(\mathrm{z}) \mathrm{s}}{\alpha}, \quad \mathrm{a} \in \mathrm{R} \backslash\{0\}
$$

Using the hypothesis (28), we obtain:

$$
\begin{gathered}
\Psi\left(\mathrm{q}(\mathrm{z}), \mathrm{zq}^{\prime}(\mathrm{z}) ; \mathrm{z} \in \mathrm{U}\right) \subset \Omega=\left\{\mathrm{w} \in \mathbb{C}: \Re(\mathrm{w})>-\frac{\delta(1-\gamma)}{2 \alpha}\right\} \\
\text { Now, for all real } \mathrm{r}_{2}, \mathrm{~s}_{1} \leq-\frac{1+\mathrm{r}_{2}^{2}}{2} \text {, we have: } \\
\Re\left\{\Psi\left(\mathrm{ir}_{2}, \mathrm{~s}_{1}\right)\right\}=\frac{\mathrm{s}_{1}(1-\gamma) \Re\{\Phi\}}{\alpha} \\
\leq-\frac{\delta(1-\gamma)\left(1-\mathrm{r}_{2}^{2}\right)}{2 \alpha} \leq-\frac{\delta(1-\gamma)}{2 \alpha}
\end{gathered}
$$

This shows that $\Re\left\{\Psi\left(\mathrm{ir}_{2}, \mathrm{~s}_{1}\right)\right\} \notin \Omega$ for each $\mathrm{z} \in \mathrm{U}$. Hence by Lemma $A$, we have $\Re\{q(z)\}>0, z \in U$. This proves (32). The proof of (33) follows by using (32) and (33) in the identity:

$$
\begin{aligned}
& \Re\left\{\frac{I^{k} L(\alpha+1, \beta) f(z)}{I^{k} L(\alpha+1, \beta) g(z)}\right\} \\
& =\Re\left\{\frac{I^{k} L(\alpha+1, \beta) f(z)}{I^{k} L(\alpha+1, \beta) g(z)}-\frac{I^{k} L(\alpha, \beta) f(z)}{I^{k} L(\alpha, \beta) g(z)}\right\} \\
& +\Re\left\{\frac{I^{k} L(\alpha, \beta) f(z)}{I^{k} L(\alpha, \beta) g(z)}\right\}
\end{aligned}
$$

This completes the proof of Theorem 3 .

\section{DISCUSSION}

By putting some values in Corollaries 2 and 3 and Theorem 3 we can obtain the following:

- Putting $\lambda=1$ and $\alpha, \beta>0$ in Corollary 2, we have $\Re\left\{z^{p} I^{k} L(\alpha+1, \beta) f(z) \cdot\left(z^{p} I^{k} L(\alpha, \beta) f(z)\right)^{\mu-1}\right\}>\gamma$ implies $\Re\left\{z^{p} I^{k} L(\alpha, \beta) f(z)\right\}^{\mu}>\frac{2 a \gamma+\Re\{\lambda\}}{2 a+\Re\{\lambda\}}, z \in U$

- For $\lambda \in \mathbb{C} \backslash\{0\}$ with $\Re\{\lambda\}>0, \mu=1$ and $\alpha$, $\beta>0$ in Corollary 2, we have $\Re\left\{(1-\lambda) z^{p} I^{k} L(\alpha, \beta) f(z)+\lambda\left(z^{p} I^{k} L(\alpha+1, \beta) f(z)\right)\right\}>\gamma$, $(0 \leq \gamma<1)$ implies $\Re\left\{\mathrm{z}^{\mathrm{p}} \mathrm{I}^{\mathrm{k}} \mathrm{L}(\alpha, \beta) \mathrm{f}(\mathrm{z})\right\}>\frac{2 \mathrm{a} \gamma+\Re\{\lambda\}}{2 \mathrm{a}+\Re\{\lambda\}}$, $\mathrm{z} \in \mathrm{U}$ Choosing $\mathrm{k}=0$ and $\alpha=\beta=\mathrm{p}$ appropriately in Corollary 2 , we obtain the following results 
- For $\lambda=1, \mathrm{k}=0$ and $\alpha=\beta=\mathrm{p}$ in Corollary 2, we have $\Re\left\{\frac{\mathrm{zf}^{\prime}(\mathrm{z})}{\operatorname{pf}(\mathrm{z})}\left(\mathrm{z}^{\mathrm{p}} \mathrm{f}(\mathrm{z})\right)^{\mu}\right\}>\gamma,(0 \leq \gamma<1, \mu>0, \mathrm{p} \in \mathrm{N})$ implies $\Re\left\{\mathrm{z}^{\mathrm{p}} \mathrm{f}(\mathrm{z})\right\}^{\mu}>\frac{2 \mathrm{p} \gamma \mu+1}{2 \mathrm{p} \mu+1}, \mathrm{z} \in \mathrm{U}$

- For $\lambda \in \mathbb{C} \backslash\{0\}$ with $\mathfrak{R}\{\lambda\}>0, \mu=1, \mathrm{k}=0$ and $\alpha=\beta=\mathrm{p}$ in Corollary 2, we have $\Re\left\{(1-\lambda) \mathrm{z}^{\mathrm{p} f}(\mathrm{z})+\frac{\lambda}{\mathrm{p}}\left(\mathrm{z}^{\mathrm{p}+1} \mathrm{f}^{\prime}(\mathrm{z})\right)\right\}>\gamma, \quad(0 \leq \gamma<1, \quad \mu>0$, $\mathrm{p} \in \mathrm{N}$ ) implies $\Re\left\{\mathrm{z}^{\mathrm{p}} \mathrm{f}(\mathrm{z})\right\}>\frac{2 \mathrm{p} \gamma+\Re\{\lambda\}}{2 \mathrm{p}+\Re\{\lambda\}}, \mathrm{z} \in \mathrm{U}$

- Replacing $f(z)$ by $-\frac{z^{\prime}(z)}{p}$ in the result (ii), we have $-\Re\left\{(1-\lambda) \frac{\mathrm{z}^{\mathrm{p}+1}}{\mathrm{p}} \mathrm{f}(\mathrm{z})+\frac{\lambda}{\mathrm{p}^{2}}\left(\mathrm{z}^{\mathrm{p}+2} \mathrm{f}^{\prime \prime}(\mathrm{z})\right)\right\}>\gamma \quad$ implies $-\Re\left\{\frac{z^{p+1}}{p} f^{\prime}(z)\right\}>\frac{2 p \gamma+\Re\{\lambda\}}{2 p+\Re\{\lambda\}}, z \in U$

- For $\lambda \in \Re$ with $\lambda \geq 1 ; \quad \mu=1, \quad \mathrm{k}=0 \quad$ and $\alpha=\beta=p$ in Corollary 2, we have $\Re\left\{(1-\lambda) \mathrm{z}^{\mathrm{p}} \mathrm{f}(\mathrm{z})+\frac{\lambda}{\mathrm{p}}\left(\mathrm{z}^{\mathrm{p}+1} \mathrm{f}^{\prime}(\mathrm{z})\right)\right\}>\gamma \quad(0 \leq \gamma<1, \quad \mathrm{z} \in \mathrm{U})$ implies $\Re\left\{\mathrm{z}^{\mathrm{p}} \mathrm{f}(\mathrm{z})\right\}>\frac{(2 \mathrm{p}+1) \gamma+\lambda-1}{2 \mathrm{p}+\lambda}$

Next by choosing values in Corollary 3 we have the following remarks:

- We note that if $\lambda=\mu>0, \alpha=\beta=p$ and $k=0$ in Corollary 3 , that is:

$$
\Re\left\{(1-\lambda)\left(z^{\mathrm{p}} \mathrm{f}(\mathrm{z})\right)^{\lambda}+\frac{\lambda \mathrm{z}^{\mathrm{p}+1}}{\mathrm{p}} \mathrm{f}^{\prime}(\mathrm{z})\left(\mathrm{z}^{\mathrm{p}} \mathrm{f}(\mathrm{z})\right)^{\lambda-1}\right\}>\gamma
$$

Then (20) implies that:

$$
\Re\left\{z^{\mathrm{p}} \mathrm{f}(\mathrm{z})\right\}^{\lambda}>\frac{2 \mathrm{p} \gamma+1}{2 \mathrm{p}+1}, \quad(\mathrm{z} \in \mathrm{U})
$$

whereas if $f(z) \in \sum_{p}$ satisfies the condition (33) then by using Theorem 2, we have:

$\Re\left\{z^{\mathrm{p}} \mathrm{f}(\mathrm{z})\right\}^{\lambda}>2(1-\ell \mathrm{n} 2) \gamma+(2 \ell \mathrm{n} 2-1)$

which is better than (34)

- We observe that if $\lambda \in \Re$ satisfying $\lambda \geq 0$ and $\mathrm{k}(\mathrm{z})=\frac{\mathrm{I}^{\mathrm{k}} \mathrm{L}(\alpha+1, \beta) \mathrm{f}(\mathrm{z})}{\mathrm{I}^{\mathrm{k}} \mathrm{L}(\alpha+1, \beta) \mathrm{g}(\mathrm{z})}+\left(\frac{1}{\lambda}-1\right) \frac{\mathrm{I}^{\mathrm{k}} \mathrm{L}(\alpha, \beta) \mathrm{f}(\mathrm{z})}{\mathrm{I}^{\mathrm{k}} \mathrm{L}(\alpha, \beta) \mathrm{g}(\mathrm{z})} \quad$ then from Theorem 1 (for $\mu=1$ ), we have $\Re\{k(z)\}>\frac{\gamma}{\lambda}$ Implies:

$$
\Re\left\{\frac{I^{k} L(\alpha, \beta) f(z)}{I^{k} L(\alpha, \beta) g(z)}\right\}>\frac{2 \alpha \gamma+\lambda \delta}{2 \alpha+\lambda \delta}
$$

Whenever:

$$
\Re\left\{\frac{\mathrm{I}^{\mathrm{k}} \mathrm{L}(\alpha, \beta) \mathrm{g}(\mathrm{z})}{\mathrm{I}^{k} \mathrm{~L}(\alpha+1, \beta) \mathrm{g}(\mathrm{z})}\right\}>\delta, \quad 0 \leq \delta<1
$$

Let $\lambda \rightarrow+\infty$, then from (35), we have $\Re\{\mathrm{k}(\mathrm{z})\}>0$ implies:

$$
\Re\left\{\frac{I^{\mathrm{k}} \mathrm{L}(\alpha, \beta) \mathrm{f}(\mathrm{z})}{\mathrm{I}^{\mathrm{k}} \mathrm{L}(\alpha+1, \beta) \mathrm{g}(\mathrm{z})}\right\} \geq 1, \mathrm{z} \in \mathrm{U}
$$

Whenever:

$$
\Re\left\{\frac{\mathrm{I}^{\mathrm{k}} \mathrm{L}(\alpha, \beta) \mathrm{g}(\mathrm{z})}{\mathrm{I}^{\mathrm{k}} \mathrm{L}(\alpha+1, \beta) \mathrm{g}(\mathrm{z})}\right\}>\delta,(0 \leq \delta<1, \mathrm{z} \in \mathrm{U})
$$

Finally, we have the following:

- Putting $\alpha=\beta=\mathrm{p}, \mathrm{k}=0$ and $\mathrm{g}(\mathrm{z})=\frac{1}{\mathrm{z}^{\mathrm{p}}}$ in Theorem 3 for all $\mathrm{z} \in \mathrm{U}$, we obtain:

$$
\Re\left\{z^{p} f(z)+\frac{z^{p+1}}{p} f^{\prime}(z)\right\}>-\frac{\delta(1-\gamma)}{2 p}
$$

implies $\Re\left\{z^{p} f(z)\right\}>\gamma$ and:

$$
\mathfrak{R}\left\{2 \mathrm{z}^{\mathrm{p}} \mathrm{f}(\mathrm{z})+\frac{\mathrm{z}^{\mathrm{p}+1}}{\mathrm{p}} \mathrm{f}^{\prime}(\mathrm{z})\right\}>\frac{(2 \mathrm{p}+\delta) \gamma-\delta}{2 \mathrm{p}}
$$

- For $\alpha=p+1, \beta=p, k=0$ and $g(z)=\frac{1}{z^{p}} \quad$ in Theorem 3 for all $\mathrm{z} \in \mathrm{U}$, we have $\Re\left\{\mathrm{z}^{\mathrm{p}} \mathrm{I}^{\mathrm{k}} \mathrm{L}(\mathrm{p}+2, \mathrm{p}) \mathrm{f}(\mathrm{z})-\mathrm{z}^{\mathrm{p}} \mathrm{I}^{\mathrm{k}} \mathrm{L}(\mathrm{p}+1, \mathrm{p}) \mathrm{f}(\mathrm{z})\right\}>-\frac{(1-\gamma) \delta}{2(\mathrm{p}+1)}$ $(0 \leq \gamma<1,0 \leq \delta<1)$, implies $\Re\left\{z^{\mathrm{p}} \mathrm{I}^{\mathrm{k}} \mathrm{L}(\mathrm{p}+1, \mathrm{p}) \mathrm{f}(\mathrm{z})\right\}>\gamma$ and $\Re\left\{\mathrm{z}^{\mathrm{p}} \mathrm{I}^{\mathrm{k}} \mathrm{L}(\mathrm{p}+2, \mathrm{p}) \mathrm{f}(\mathrm{z})\right\}>\frac{(2(\mathrm{p}+1)+\delta) \gamma-\delta}{2(\mathrm{p}+1)}$ 


\section{CONCLUSION}

The operator defined was motivated by various work studied earlier by the authors (Joshi and Srivastava, 1999; Liu and Srivastava, 2001; 2004a; 2004b). This operator can be generalized further and many other results such as the coefficient estimates and distortion theorem can be obtained.

\section{ACKNOWLEDGEMENT}

The study here is fully supported by EScienceFund grant: 04-01-02-SF0425, MOSTI, Malaysia.

\section{REFERENCES}

Aouf, M.K., 1993. New criteria for multivalent meromorphic starlike functions of order $\alpha$. Proc. Jap. Acad. Ser. A., 69: 66-70. DOI: 10.3792/pjaa.69.66

Aouf, M.K. and H.M. Hossen, 1993. New criteria for meromorphic p-valent starlike functions. Tsukuba J. Math., 17: 481-486. http://ci.nii.ac.jp/naid/110000027376/

Aouf, M.K. and H.M. Srivastava, 1997. A new criteria for meromorphic p-valent convex functions of order $\alpha$. Math. Sci. Res. Hot-Line, 1: 7-12. http://ear.ro/3brevist/rv39/aouf-al_oboudi-haidan.pdf

Cho, N.E. and I.H. Kim, 2007. Inclusion properties of certain classes of meromorphic functions associated with the generalized hypergeometric function. Applied Math. Comput., 187: 115-121. http://cat.inist.fr/?aModele $=$ afficheN\&cpsidt $=1879$ 6492

Dziok, J. and H.M. Srivastava, 2002. Some subclasses of analytic functions with fixed argument of coefficients associated with the generalized hypergeometric function. Adv. Stud. Contemp. Math. Kyungshang, 5: 115-125.

Dziok, J. and H.M. Srivastava, 2003. Certain subclasses of analytic functions associated with the generalized hypergeometric function. Trans. Spec. Funct., 14: 7-18.

Ghanim, F. and M. Darus, 2009a. Linear operators associated with subclass of hypergeometric meromorphic uniformly convex functions. Acta Univ. Apulensis, 17: 49-60. http://www.emis.ams.org/journals/AUA/acta17/pap er\%2006-17-2009.pdf

Ghanim, F. and M. Darus, 2009b. Some properties of certain subclass of meromorphically multivalent functions defined by liner operator. Am. J. Sci. Res., $\quad$ 2: 52-59. http://www.eurojournals.com/ajsr_2_06.pdf
Joshi, S.B. and H.M. Srivastava, 1999. A certain family of meromorphically multivalent functions. Comput. Math. Applied, 38: 201-211. http://direct.bl.uk/bld/PlaceOrder.do?UIN=069758 $150 \&$ ETOC $=$ RN \& from $=$ searchengine

Kukarni, S.R., U.H. Naik and H.M. Srivastava, 1998. A certain class of meromorphically p-valent quasiconvex functions. Pan Am. Math. J., 8: 57-64. http://direct.bl.uk/bld/PlaceOrder.do?UIN=039984 210\&ETOC $=\mathrm{RN} \&$ from $=$ searchengine

Liu, J.L., 2003. A linear operator and its applications on meromorphic p-valent functions. Bull. Inst. Math. Acad. Sin., 31: 23-32. http://direct.bl.uk/bld/PlaceOrder.do?UIN=131276 047\&ETOC $=$ RN\& from $=$ searchengine

Liu, J.L. and H.M. Srivastava, 2001. A linear operator and associated families of meromorphically multivalent functions. J. Math. Anal. Applied, 259: 566-581.

http://cat.inist.fr/?aModele $=$ afficheN\&cpsidt $=1140$ 378

Liu, J.L. and H.M. Srivastava, 2004a. Certain properties of the Dziok-Srivastava operator. Applied Math. Comput., 159: 485-493. http://cat.inist.fr/?aModele $=$ afficheN\&cpsidt $=1652$ 9743

Liu, J.L. and H.M. Srivastava, 2004b. Classes of meromorphically multivalent functions associated with the generalized hypergeometric function. Math. Comput. Model., 39: 21-34. http://cat.inist.fr/?aModele $=$ afficheN\&cpsidt $=1541$ 9538

Miller, S.S. and P.T. Mocanu, 1978. Second order differential inequalities in the complex plane. J. Math. Anal. Applied, 65: 289-305. http://www.springerlink.com/content/g2045580431 81371/fulltext.pdf?page $=1$

Mogra, M.L., 1990a. Meromorphic multivalent functions with positive coefficients I. Math. Jap., 35: 1-11.

Mogra, M.L., 1990a. Meromorphic multivalent functions with positive coefficients II, Math. Jap., 35: 1089-1098.

Owa, S., H.E. Darwish and M.K. Aouf, 1997. Meromorphic multivalent functions with positive and fixed second coefficients. Math. Jap., 46: 231-236. http://direct.bl.uk/bld/PlaceOrder.do?UIN=035350 $275 \&$ ETOC $=$ RN\& from $=$ searchengine

Ponnusamy, S., 1995. Differential subordination and Bazilevic functions. Proc. Indian Acad. Sci. Math. Sci., $\quad 105:$ 169-186. http://www.springerlink.com/content/c71621053lj2 $74 \mathrm{xp} /$ 
Srivastava, H.M. and S. Owa, 1992. Current Topics in Analytic Function Theory. World Scientific, Singapore, New Jersey, London, Hong Kong, ISBN: 9810209320, pp: 456.

Uralegaddi, B.A. and C. Somanatha, 1992. Certain classes of meromorphic multivalent functions. Tamkang J. Math., 23: 223-231. http://www.mathnet.or.kr/mathnet/thesis_content.p hp?no=270081

Uralegaddi, B.A. and C. Somanatha, 1991. New Criteria for meromorphic starlike univalent functions. Bull. Aust. Math. Soc., 43: 137-140. http://cat.inist.fr/?aModele $=$ afficheN\&cpsidt $=5529$ 155
Whittaker, E.T. and G.N. Watson, 1927. A Course on Modern Analysis: An Introduction to the General Theory of Infinite Processes and of Analytic Functions; with an Account of the Principal Transcendental Functions. 4th Edn., Cambridge University Press, Cambridge, ISBN: 0521588073, pp: 616.

Yang, D.G., 1996. On a class of meromorphic starlike multivalent functions. Bull. Inst. Math. Acad. Sinica, 24: 151-158. http://direct.bl.uk/bld/PlaceOrder.do?UIN=013383 030\&ETOC $=$ RN\& from $=$ searchengine

Yang, D.G., 1995. On new subclasses of meromorphic p-valent functions. J. Math. Res. Exposit., 15: 7-13. 